\title{
Développements récents d'une méthode de prévision de l'érosion de cavitation
}

\author{
A. Amblard \& A. Bonazzi Alsthom CERG, Le Pont de Claix \\ Y. Lecoffre Bassin des Carènes, Le Val de Reuil
}

\section{Introduction}

La recherche d'une grande compacité des machines hydrauliques conduit fréquemment à utiliser des vitesses de fluide importantes. Ainsi on peut citer certaines pompes ou turbines-pompes de haute chute dont les vitesses relatives peuvent atteindre 35 à $70 \mathrm{~m} / \mathrm{s}$. Ces valeurs élevées de la vitesse et la faible valeur du NPSH peuvent entraîner un fonctionnement en régime de cavitation, cette cavitation apparaissant sur le bord d'attaque des aubes. Actuellement, il n'existe que des méthodes qualitatives (par exemple, enlèvement de peinture) permettant de prédire si le fonctionnement est érosif et où se situent les zones d'impacts de l'érosion de cavitation.

Depuis 1982, à l'initiative du CERG, de l'EDF, de l'IMG et de la DRET, un programme de recherche a été mis en place afin de mieux connaitre les lois générales régissant l'érosion de cavitation et de définir une méthode pratique de prédiction quantitative de l'érosion de cavitation produite au réel à partir d'essais de courte durée sur modèle.

La démarche retenue repose sur l'hypothèse implicitement admise de la similitude géométrique des écoulements modèle et prototype tant sur l'écoulement global que sur l'écoulement local, c'est-à-dire la formation et le collapse des structures de vapeur. Les conditions modèle sont une combinaison partielle ou totale des paramètres suivants : vitesse, échelle, fluide et matériau. Le présent article examine principalement l'influence de l'air dissous sur l'érosion de cavitation.

\section{Rappel des bases de la méthode}

La méthode consiste à placer des échantillons polis dans un écoulement cavitant pendant un temps relativement court. L'agressivité de la cavitation est alors révélée par lecture des empreintes isolées, causées par les impacts, à la surface de l'échantillon.



1. Tracé hydraulique de la veine d'essais (Echelle 1, Veine 1/3).

\section{Recent developments concerning a method for forecasting cavitation erosion.}

In the first part, the authors refer to the laws of similarity of a quantitative forecast method concerning erosion produced by a cavitation flow and give the results of validation tests carried out in various laboratories (Alsthom-CERG, EDF and the Grenoble Institute of Mechanics). The second part relates to the analysis on the basis of the test results of the effect of dissolved air on erosion produced by a pocket cavitation. The third part gives an account of the steps taken concerning the continuation of the development and the use of the method, determination of impact histograms by optical measurement and the development of a controlled simulation measuring device of cavitation erosion impacts. 


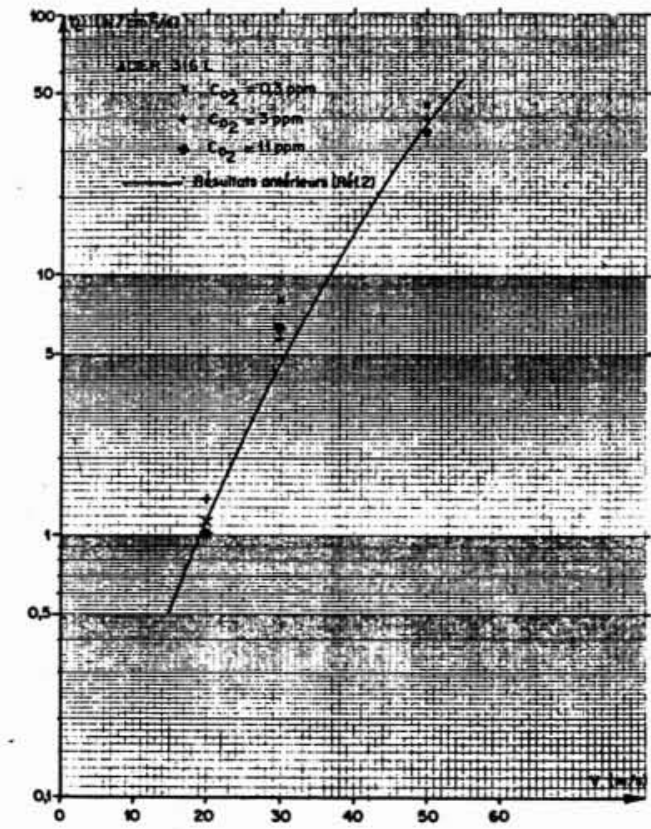

2. Influence de l'air dissous sur l'érosion de cavitation. Densité d'impacts en fonction de la vitesse d'écoulement.



3. CAVERSIM : Conception veine fixe.

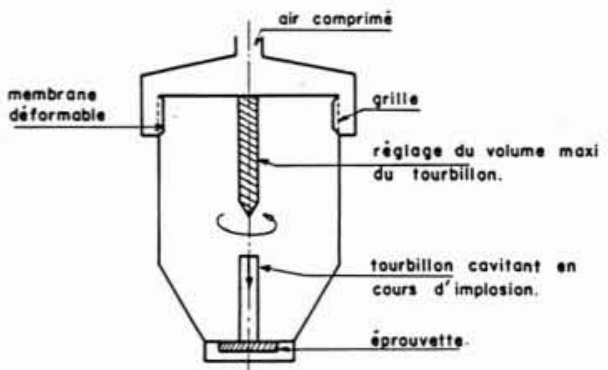

4. CAVERSIM : Conception veine tournante.

\section{Etude d'influence de l'air dissous sur l'érosion de cavitation}

Dans la veine d'essais (fig. 1), l'écoulement présente un décollement au col du venturi, la cavitation est initiée en ce point et se présente sous forme d'une poche de vapeur attachée au point de décollement. A l'aval il se produit un entraînement plus ou moins important de structures de vapeur. Cette phase du phénomène consiste en une vidange partielle de la poche.

\section{a. Diffusion gazeuse}

La poche de vapeur est alimentée en air dissous par diffusion gazeuse au travers de l'interface. Le débit masse de l'air s'exprime par la loi de Fick:

$$
\frac{\mathrm{d} m}{\mathrm{~d} t}=K_{L} \cdot A \cdot H\left(P_{\text {sat }}-P_{g}\right)
$$

$K_{L} \quad$ : coefficient de transfert de l'interface $(\mathrm{m} / \mathrm{s})$,

$A$ : surface de transfert de l'interface $\left(\mathrm{m}^{2}\right)$,

$H \quad$ : constante de Henry $\left(\mathrm{kg} / \mathrm{Pa} \cdot \mathrm{m}^{3}\right)$,

$P_{\text {sat }} \quad$ : pression de saturation du liquide (concentration en air dissous) $(\mathrm{Pa})$,

$P_{g} \quad$ : pression du gaz dans la poche $(\mathrm{Pa})$.

Si l'on considère la forme géométrique de la poche, on peut écrire que la surface interfaciale de la poche est égale à : $A=p . \ell$ (où $p$ est le périmètre de la poche et $\ell$ est la longueur de la poche) d'où :

$$
\frac{\mathrm{d} m}{\mathrm{~d} t}=K_{L} \cdot H \cdot \ell \cdot p P_{\text {sat }} \text { pour } P_{\text {sat }} \gg P_{g} .
$$

\section{b. Entraînement du gaz}

Ce gaz transmis à la poche par diffusion gazeuse est entraîné à l'aval selon un mécanisme complexe dans lequel interviennent la géométrie de la poche, les effets visqueux, les effets dus à la tension superficielle et les champs de vitesse et de pression.

Ce débit de gaz entraîné s'exprime par :

$$
\frac{\mathrm{d} m}{\mathrm{~d} t}=\rho_{g} \cdot C_{q} \cdot h \cdot p V_{c}=\rho_{g_{\mathrm{atm}}} \cdot \frac{p_{g}}{P_{\mathrm{atm}}} \cdot C_{q} \cdot h \cdot p \cdot V_{c}
$$

où $C_{q}$ est la proportion volumique de la poche qui est vidangée et $h$ est la hauteur de la poche.

A partir des expressions (1) et (2), on exprime la pression de gaz, $P_{g}$, entraînée à l'aval de la poche dans les structures de vapeur:

$$
P_{g} \frac{K_{L}}{C_{q}} \cdot \frac{H \cdot P_{\text {sat }}}{\rho_{\text {atm }} V_{c}} \cdot \frac{\ell}{h} \cdot P_{\text {atm }} .
$$

Ainsi, pour une géométrie de poche donnée (veine 1/3) (fig. 1) dont les caractéristiques sont les suivantes :

$$
\begin{aligned}
& K_{L}=10^{-3} \mathrm{~m} / \mathrm{s}, H=0,5 \cdot 10^{-6} \mathrm{~kg} / \mathrm{m}^{3} \mathrm{~Pa}, \\
& \ell=30 \mathrm{~mm}, h=3 \mathrm{~mm}, \\
& C_{q}=0,2 \mathrm{à} 0,3, \\
& V_{c}=20 \mathrm{~m} / \mathrm{s} \text { (valeur la plus défavorable), } \\
& P_{\text {atm }}=10^{5} \mathrm{~Pa}, \rho_{g_{\text {atm }}}=1,3 \mathrm{~kg} / \mathrm{m}^{3} .
\end{aligned}
$$

On aboutit aux valeurs de pression de gaz suivantes :

- Si $\mathrm{C}_{\mathrm{O}_{2}}=10 \mathrm{ppm}\left(P_{\text {sat }}=10^{5} \mathrm{~Pa}\right)$ alors $P_{g} \simeq 14 \mathrm{~Pa}$.

- $\mathrm{Si} \quad \mathrm{C}_{\mathrm{O}_{2}}=0,1 \mathrm{ppm} \quad\left(P_{\text {sat }}=10^{3} \mathrm{~Pa}\right) \quad$ alors $P_{g} \simeq 0,14 \mathrm{~Pa}$.

Ces valeurs nous permettent d'analyser l'influence de ces pressions partielles sur le collapse d'une bulle sphérique. Ainsi si l'on prend le modèle de Hickling et Plesset, il apparaît qu'une variation de pression partielle $P_{g}$ de 0 à $20 \mathrm{~Pa}$ modifie très faiblement la vitesse de paroi de la bulle, ceci jusqu'à la valeur de $R / R_{0} \simeq 0,02\left(R_{0}\right.$ : rayon initial de la bulle).

Des essais ont été réalisés en eau à trois valeurs de concentration en oxygène dissous $0,1,3$ et $11 \mathrm{ppm}$ (correspondant aux trois pressions de saturation 0,01 , 
0,3 et 1 bar) et pour trois vitesses d'écoulement $V=20$, 30 et $50 \mathrm{~m} / \mathrm{s}$. Cette campagne d'essais a permis de vérifier ces hypothèses et de montrer que dans ces conditions de cavitation, l'air dissous n'influe pas sur l'érosion (fig. 2).

Ces résultats peuvent étonner étant donné qu'il est plus ou moins admis dans la littérature que la teneur en air dissous a une forte influence sur l'érosion de cavitation. Ces différentes études doivent être interprétées avec précaution :

- dans de nombreux cas, la modification en air dissous a une forte influence sur l'air libre (microbulles) et en conséquence, sur la géométrie de cavitation,

- pour ce qui est de l'air libre, il est nécessaire d'injecter des quantités importantes si l'on veut réduire l'érosion. Ainsi, il semble nécessaire d'injecter un débit d'air de $1 \%$ du débit d'eau pour réduire le taux d'érosion d'un venturi à 60 à $80 \mathrm{~m} / \mathrm{s}$. Un tel taux de vide est rarement rencontré industriellement.

Ainsi les résultats obtenus lors de l'étude réalisée au CERG montrent clairement que dans le cas d'une cavitation à poche, on peut réaliser des essais d'érosion simulant les conditions réelles, quelle que soit la teneur en gaz dissous dans la gamme classique d'utilisation.

\section{Développement et application de la méthode de prévision}

Tous ces résultats nous confortent dans la démarche entreprise sur la faisabilité d'une méthodologie de prévision de l'érosion de cavitation. Le développement de cette méthode nécessite de vérifier la transposition des histogrammes, de mettre au point une méthode automatique de dépouillement et de disposer d'un appareil destiné à reproduire des impacts contrôlés d'érosion (Le Caversim).

L'appareil Caversim (simulation d'érosion de cavitation) est destiné à reproduire des impacts d'érosion de cavitation (du type vortex cavitant) en maittrisant l'intensité, la taille et la localisation de ces impacts. Deux concepts d'appareil sont envisagés :

\subsection{Concept veine fixe (fig. 3)}

Il s'agit d'étudier et d'améliorer les conditions d'écoulement de la veine tourbillon existante. Le principe de fonctionnement de la veine restant le même.

L'entrée tangentielle et la sortie axiale de la veine crée un écoulement vortex dont le cœur est en rotation solide. L'interruption brutale du débit au moyen de l'obturateur rotatif à l'amont de la veine crée un coup de bélier et l'ouverture d'un vortex de vapeur situé dans la zone en rotation solide. Il se produit alors une propagation d'ondes et une inversion du débit de la colonne liquide venant refermer le vortex par inertie. Bien que tous ces phénomènes soient complexes, une analyse détaillée doit nous permettre de contrôler au mieux le processus.

Ainsi l'étude du concept veine fixe, portant essentiellement sur les paramètres géométriques de la veine et sur le fonctionnement de l'obturateur, doit nous permettre de maîtriser la symétrie, le volume du noyau de vapeur ainsi que la vitesse de collapse.

\subsection{Concept veine tournante (fig. 4)}

Cette nouvelle conception doit permettre de reproduire l'écoulement en rotation solide existant au centre de la veine tourbillon. Pour cela, on remplit préalablement la veine d'un liquide dépourvu de gaz dissous ; elle est alors mise en rotation par un moteur électrique et crée un écoulement en rotation solide où les vitesses tangentielles sont proportionnelles au rayon.

Il s'agit alors d'imposer des conditions limites connues et réglables dans les phases d'ouverture et de fermeture du vortex de vapeur. Ainsi, on provoque l'ouverture du vortex de vapeur sur l'axe de rotation de l'appareil par accroissement du volume interne de la veine au moyen d'une membrane souple pilotée par mise au vide. On referme ensuite le vortex brutalement en imposant sur la membrane une pression donnée (analogue à une pression à l'infini en écoulement). Le collapse doit alors créer un impact d'érosion semblable à celui de la veine fixe.

L'étude du concept veine tournante doit permettre de définir les dispositifs de remplissage, de mise en rotation de la veine, de la création, du contrôle et du collapse du noyau de vapeur ainsi que la position du collapse sur l'échantillon.

Pour les deux concepts envisagés, le CERG doit également définir l'ensemble des moyens de mesure : pression, température, vitesse, concentration en gaz dissous et en germes ainsi que les dispositifs de visualisation.

A l'issue de ces études, le CERG choisira le principe de fonctionnement du Caversim afin de réaliser l'étude d'exécution de l'appareil ainsi que l'analyse des perspectives d'exploitation.

Pour ce qui est de l'exploitation d'un tel appareil, le Caversim doit permettre, dans le domaine de l'érosion de cavitation, d'appréhender bon nombre des aspects du problème, notamment :

- dans le domaine de l'hydrodynamique : établissement et vérification des lois de similitude du modèle proposé, reproduction d'histogrammes prototype...

- dans le domaine de la mécanique du solide : étude de l'interaction fluide matériau,

- dans le domaine de la science des matériaux : étude du comportement des matériaux à l'érosion avec une gamme plus vaste et mieux maîtrisée des intensités érosives.

\section{Conclusion}

Les résultats des études antérieures ont permis de vérifier le bien-fondé du modèle proposé. Ainsi les mesures de densités d'impacts par comptage manuel sous observation microscopique ont permis de valider les lois de transposition prédites pour les effets d'échelles, le changement de fluide et de matériau.

Une étude supplémentaire, réalisée en eau sur le même moyen d'essais, a permis de mettre en évidence 
qu'une variation de la teneur en air dissous de 0,1 à 11 ppm n'entraîne pas de variation notable de l'érosion de cavitation produite par une cavitation à poche.

Ce résultat montre que l'on peut réaliser des essais d'érosion en laboratoire industriel simulant les conditions réelles et ceci quelle que soit la teneur en gaz dissous.

$\mathrm{Au}$ vu de ces résultats qui mettent en évidence la faisabilité d'une méthode de prévision quantitative de l'érosion de cavitation, un programme d'étude a été engagé par le CERG, la DRET, l'EDF et l'IMG dont les objectifs sont :

- d'une part de valider les lois de transposition en déterminant les histogrammes d'impacts des échantillons par la méthode optique du LOBE,

- et d'autre part, d'étudier et de développer l'appareil Caversim dont le but est de générer des impacts de vortex cavitant en maitrisant la taille et la vitesse de collapse, le développement d'un tel appareil permettant d'aborder de nombreux aspects de l'érosion (hydrodynamique, interaction fluide-matériau, étude de comportement de matériau).

Adresses des auteurs :

$\begin{array}{ll}\text { Messieurs A. Amblard } & \text { Monsieur Y. Lecoffre } \\ \text { A. Bonazzi } & \text { Bassin des carènes } \\ \text { Alsthom CERG } & \text { Chaussée du Vexin } \\ \text { Voie privée Rhône-Poulenc } & 27100 \text { Val de Reuil } \\ 38800 \text { Le Pont de Claix } & \text { Tél. : } 32597800 \\ \text { Tél. : (16) } 76409040 & \end{array}$

\section{Discussion}

Président : M. P. LAvY

M. BINDEL : Concernant l'influence de l'air dissous, je suis étonné qu'il $\mathrm{y}$ ait une diffusion gazeuse au travers de l'interface de la poche de vapeur qui se forme au point de décollement au col du venturi car il s'agit d'un phénomène très lent, beaucoup plus long en tout cas que la durée de vie de la poche. La quantité d'air contenu dans la poche dépend certainement de la teneur totale de l'air mais y a-t-il vraiment un phénomène dynamique de diffusion? Jaimerais donc savoir quel est le résultat des calculs faits par les auteurs à partir de la loi de Fick.

M. AMBLARD: Le calcul de la quantité d'air susceptible de migrer à partir du fluide supposé sursaturé donne des concentrations beaucoup trop faibles pour qu'il y ait influence de l'air dissous sur l'érosion. C'est un problème de temps caractéristique.

M. BINDEL : Cela confirme que le phénomène est très lent. Sur un autre plan, je voudrais faire remarquer à tous les auteurs que le mot " collapsus " figure dans le dictionnaire français alors que le mot "collapse " utilisé par la plupart des gens est américain.

Mme NIENALTOWSKA : Avez-vous constaté la présence d'air au cœur du tourbillon dans la cuve Vortex pour des temps de fonctionnement très courts?

M. AMBLARD : Pendant quelques minutes il n'y a pas de gaz.

M. BONNIN : La bonne confrontation entre les érosions obtenues avec trois liquides aussi différents que l'eau, le sodium et le mercure, extrêmement encourageante pour la similitude choisie, me permet de féciliter les auteurs.

Néanmoins, les trois liquides, étudiés sont des «liquides froids » au sens thermodynamique, c'est-à-dire ne présentant pas, dans ce sens, de résistance appréciable au changement de phase ${ }^{\left({ }^{(}\right)}$. Il n'en serait pas de même si l'on opérait avec des « liquides chauds ", tels que eau chaude, hydrocarbure, liquides cryogéniques.
Envisagez-vous d'effectuer avec de tels liquides des essais d'érosion comparatifs avec ceux des liquides précédents?

Dans ce cas, il y aurait lieu de tenir compte des paramètres thermodynamiques de cavitation B' et B" (faisant suite au paramètre $\mathrm{B}$ de Stépanoff) ${ }^{(\cdots)}$.

Dans une certaine mesure, on pourrait alors comparer ces nouveaux essais à ceux effectués avec des liquides engazés, en substituant la diffusivité thermique à la diffusivité massique, ce qui revient à remplacer le nombre de Jakob par un nombre de Jakob généralisé $\left({ }^{* * *}\right)$, et répond partiellement à la remarque précédente faite par M. BINDEL.

M. AMBLARD : Actuellement nous n'avons pas d'étude en cours sur ce sujet.

M. AVELLAN : Il y a eu confusion au niveau de l'influence de l'air dissous entre collapsus pur et collapsus de Vortex, ce dernier étant rencontré dans la veine tourbillon où l'effet de l'air dissous est considérable. Jaimerais être éclairé sur ce point.

M. AMBLARD: C'est toujours un problème de temps caractéristique : on peut avoir un engazage progressif du Vortex.

$M$. LECOFFRE : Les expérimentations ont montré qu'il existe un seuil de saturation en air dissous au-delà duquel on a formation de bulles ce qui rend inefficace la veine tourbillon. Le problème est qu'alors la bulle reste coincée et grossit par diffusion gazeuse.

M. AVELLAN : Je serais d'accord avec vous: on voit des bulles coincées dans l'axe du vortex.

M. KARIMI: On ne peut pas dégazer tout l'air dissous dans l'eau. Il en reste toujours un peu.

M. FRUMAN: Je ne vois pas très bien comment la mise en rotation d'une cuve vous permettra d'améliorer les conditions de formation du tourbillon et contrôler sa structure et son collapsus.

M. AMBLARD: Nous sommes bien conscients que la solution proposée de veine tournante ne permet de reproduire que la partie centrale de l'écoulement dans la veine tourbillon actuelle, c'està-dire le cœur solide qui est en rotation.

M. AMBLARD (Neyrpic): Une remarque : vous avez dit que pour réduire l'érosion par cavitation, il fallait injecter un débit d'air de $1 \%$ du débit d'eau dans le circuit.

Pour que ce chiffre ne reste pas sans commentaire pour les exploitants de turbines, il faut indiquer que dans le cas des turbines Francis de grande vitesse spécifique pour réduire l'érosion de cavitation à l'entrée des aubes de la roue, le débit d'air est de 1 à $2 \%$ du débit d'eau ramené à la pression atmosphérique.

Les conditions d'expérimentation en laboratoire sur un profil de veine sont évidemment différentes des conditions industrielles.

M. MICHEL: Il me paraît important d'articuler d'une part la méthode de prévision de l'érosion de cavitation de M. KARIMI, d'autre part la méthode de similitude proposée par M. LECOFFRE.

Par exemple y a-t-il moyen de trouver par les approches métallurgiques la valeur de 1,67 que vous avez montrée dans les courbes en homothétie pour l'Inox et l'Aluminium?

Vous avez montré que l'évolution du nombre de trous en fonction de l'échelle géométrique est en $\lambda^{3}$, mais votre diagramme était en échelle logarithmique. Il y a quand même une différence me semble-t-il, et de quel coefficient?

M. AMBLARD: Il y a un facteur d'environ 1,5 entre les 2 courbes. Ce facteur rentre dans la gamme d'incertitude liée à la méthode de comptage manuel de trous sur les échantillons (20 à $30 \%$ d'incertitude). C'est la méthode de dépouillement qui est à améliorer avec le dépouillement optique.

(*) Notion de résistance diphasique - Application à la cavitation et à l'ébullition. EDF, Bulletin de la Direction des Etudes et Recherches. Série A, n 1, 1972, pp. 39-76.

$\left.{ }^{* *}\right)$ J. BONNIN. - Début de cavitation dans des liquides différents. EDF, Bulletin de la Direction des Etudes et Recherches, Série A, n 4 , 1970 , pp. 53-72.

$(* * *)$ J. BONNIN, M. REALI, L. SARdella. - Variations de valeur d'une bulle de gaz ou de vapeur. Symposium AIRH. SHF "Ecoulements diphasiques et cavitation dans les systemes de production d'Energie ". Grenoble, mars-avril 1976, pp. 17-27.

J. BonNIN. - Développements d'un nuage de cavitation. Troisième table ronde sur la rupture de veine. EDF, Bulletin de la Direction des Etudes et Recherches, Série A, n², 1977, pp. $207-$ 225 